\title{
Recovery of cognitive function soon after stroke: a study of visual neglect, attention span and verbal recall
}

\author{
DERICK T WADE, VICTORINE A WOOD, RICHARD LANGTON HEWER \\ From the Stroke Unit, Department of Neurology, Frenchay Hospital, Bristol, UK
}

SUMMARY Sixty two consecutive patients admitted to hospital and surviving 6 months were tested regularly over the first 3 months after their stroke to plot recovery in three aspects of cognition: visual neglect, assessed using a cancellation task, attention span assessed using digit span, and verbal recall, tested by counting number of words remembered from a list of 10 words. Normative data were collected for the cancellation test. Fifteen patients showed recovery from visual neglect, 10 improvement in attention span and nine improvement in verbal recall. The difficulties of measuring recovery of cognitive function after stroke are discussed.

Difficulties in defining and measuring cognitive functions may be one explanation for the relatively few studies investigating recovery and the effects of therapy in this sphere. Aphasia, one special aspect of cognitive function, is a notable exception on both counts. ${ }^{1}$ In contrast, measures of Activities of Daily Living (ADL), arm function, mood, and non-ADL activities are now becoming available and have been used to study recovery. ${ }^{2}$ One reason for the neglect of cognition may be that there are many complex measures available to trained psychologists but their use in studying groups of patients is obviously restricted, so that the few available studies are usually based on selected patients. ${ }^{3}$

We have previously studied recovery of various abilities in unselected groups of patients admitted to hospital with an acute stroke by assessing them at 2 weekly intervals or more frequently. ${ }^{4}$ We wished to extend the range of functions measured, and this paper reports our experience of using some brief simple tests of cognitive function. The primary purpose was to detect and measure change over the first 3 months.

\section{Patients and methods}

The study of recovery after stroke was based upon a 13 week follow-up of 117 consecutive admissions to Frenchay hospi-

Address for reprint requests: Dr D T Wade, Rivermead Rehabilitation Centre, Abingdon Road, Oxford OXI 4XD. UK.

Received 27 June 1986 and in revised form 11 June 1987 Accepted 15 June 1987 tal of patients with an initial diagnosis of an acute stroke. Every patient was assessed clinically to confirm the diagnosis of stroke (using the WHO definition ${ }^{5}$ ). As far as possible, each surviving patient was then tested weekly while in hospital and every 2 weeks after discharge until 13 weeks after the stroke.

The areas of cognitive function tested, and the tests used were:

1. Visual neglect: tested using a cancellation task, the patient being required to cancel all " 1 "s and " 4 "s from strings of numbers (see fig 1). This is similar to the procedure used in the Rivermead Perceptual Assessment, ${ }^{6}$ which was shown to be reliable; it used letters not numbers. Diller and Weinberg ${ }^{7}$ also used a similar task based on letters, but they apparently only had 13 control subjects (ref 8, p. 433), so we have studied 51 normal people (see later). The method of scoring is discussed later.

2. Attention: tested using the digit span, ${ }^{9}$ and scored simply as the sum of the digits recalled correctly forwards and backwards.

3. Verhal memory: tested using a list of 10 words which was read to the patient who was then asked to recall as many as possible. This is similar to the Rey Auditory-Verbal Learning Test (AVLT). ${ }^{8}$

$\begin{array}{lll}983471035635041 & 758439023875641 & 382917465960758 \\ 537260198748285 & 473820593858371 & 384958194621578 \\ 473591374653910 & 785746352601927 & 385745129109387 \\ 389152840518228 & 172935927351729 & 465725637182736 \\ 1537253948294864 & 382967109758524 & 828401831746255\end{array}$

Fig 1 Stimulus card for cancellation task. Patient to cross out "I"s and " 4 "s. 
Normative data for cancellation test

Normative data for the cancellation task were gathered from 51 people, the majority of these attending local clubs for retired or elderly people. Their performance was timed. There were 14 men and 37 women. The average (SD) age was $69 \cdot 0(11 \cdot 7)$ years, ranging from 28-87 years, median 59. Eighteen were 65 years or less, and 17 were 76 years or over.

Thirty-three people correctly cancelled all 21 numbers on the right; 15 missed one number, two missed three and one missed five. Twenty-six people correctly cancelled all 22 numbers on the left; 13 missed one number, six missed two numbers, five missed three and one missed six (she missed five on the right). Two people cancelled wrong numbers; one of these mistook a "7" for a "l" as she did not have her glasses on. Nineteen people correctly cancelled all numbers. The time taken ranged from 43-138 seconds, average (SD) 66.8 (19.9); only two people took over 110 seconds, and one of these had forgotten her glasses.

When scored as a percentage, dividing the number correctly cancelled on one side by the number correctly cancelled on the other side, all normal subjects scored between $90 \%$ and $110 \%$. This remained true whichever side's score was used as the denominator. Therefore we would suggest that the use of a relative percentage score, dividing the lower number by the higher number, is a simple way of scoring this test; the abnormal (that is, lower scoring) side should be recorded. Scored this way, results between $90 \%$ and $100 \%$ are "normal", or at least not unusual in the general elderly population. The use of alternative methods of analysing cancellation tasks is discussed later.

\section{Reliability studies}

These were conducted for all three tests on a group of 12 elderly people tested on two separate occasions 2 to 4 weeks apart. Considering the cancellation task, four subjects cancelled all digits correctly on both occasions. The other eight patients showed slight variations but there were no consistent trends. Twenty-two of the 24 results were within our previously defined "normal" limits but two subjects cancelled 19 on the right and 22 on the left on their first attempt-this is equivalent to $86 \%$. For digit span forward, Kendall's coefficient of concordance was $\mathbf{0 . 8 3}$. Four subjects had identical scores, six improved by one digit and two deteriorated by one digit. For digit span backward Kendall's coefficient of concordance was 0.78 : four were identical, three deteriorated by one point, three improved by one point, and two improved by three points. For the word learning test, Kendall's coefficient of concordance was $0 \cdot 85$ : five subjects showed no change, two deteriorated by one word, four improved by one word, and one improved by two words. We considered the test retest reliability to be acceptable.

\section{Results}

Sixty-two of the 117 patients registered form the basis of the study of recovery: 40 died before 3 months, 12 were lost to follow-up, and three were found to have tumours. The 62 patients studied comprised 28 men and 34 women; 27 had left sided weakness, 30 right sided weakness and five no unilateral weakness; their average age was $67 \cdot 7$ years (SD $12 \cdot 2$; range $26-89 ; 25$ aged less than 66,14 aged 76 or more).

In the 62 patients studied, the worst level of consciousness recorded was comatose in six, confused or sleepy in 31 , and 25 retained normal consciousness throughout. The initial clinical assessment was at a mean (SD) of $4.4(5.9)$. days after the stroke. Visual fields were tested by confrontation at the initial medical assessment: five were unassessable, 25 had a homonymous hemianopia, two had homonymous visual inattention to simultaneous stimuli, and 30 had normal visual fields.

\section{Visual neglect}

Twelve $(19 \%)$ patients could not be assessed on more than four occasions over the 3 months; eight were never able to be tested at all. A further 10 (16\%) patients had inconsistent results, sometimes neglecting the right and, at other times, neglecting the left, usually with very large fluctuations. Twenty-five $(40 \%)$ patients were "normal" throughout-their results were within our normal range on every occasion, or they were only outside normal on one occasion.

Lastly, 15 (24\%) of patients showed some recovery from their first assessment; their results are plotted in fig 2. Some of these patients also fluctuated, and the obvious deterioration seen in one patient at 76 days was associated with a further stroke. Nevertheless these curves show a more or less rapid recovery to

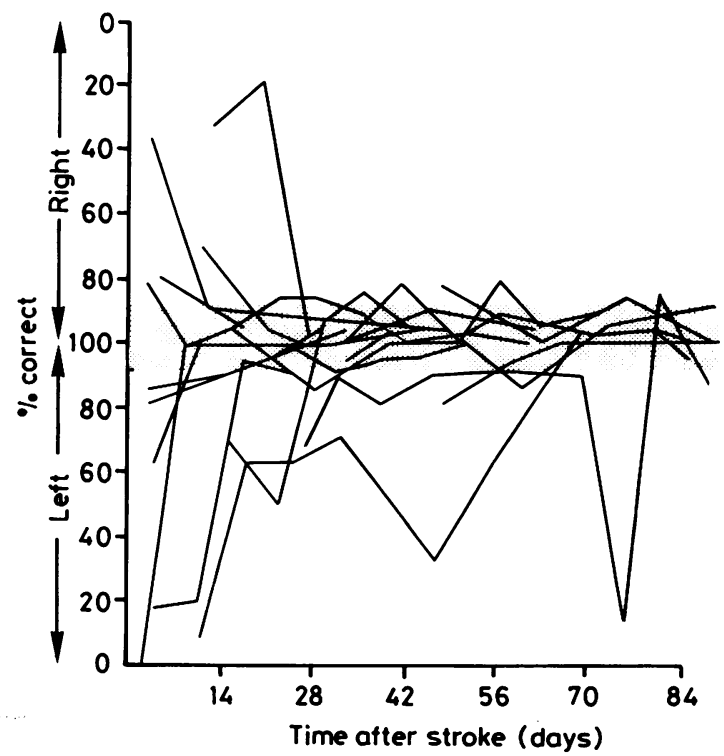

Fig 2 Recovery from visual neglect. 15 patients: percentage correct on worst side (shaded area = "normal" range). 


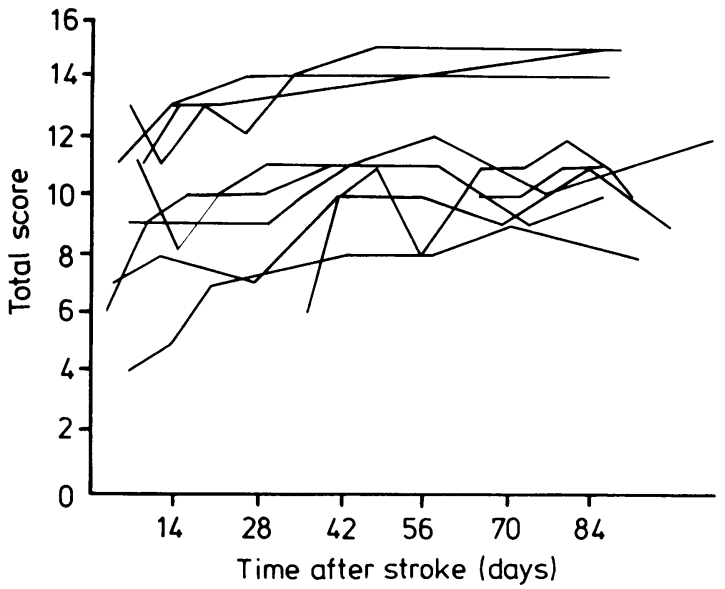

Fig 3 Recovery of digit span. 10 patients; total (forward + back) number of digits recalled.

normal over the first 70 days post-stroke. Of the 15 patients, nine had left and six right visual neglect.

The frequency of residual visual neglect at 3 months post-stroke was difficult to determine because so many patients still could not be assessed properly. Clearly the 25 normal patients, and the 15 who recovered did not have unilateral neglect. Two of the 10 patients who fluctuated had consistent neglect on their last two assessments at 11 or 12 weeks, and 13 weeks post-stroke; both neglected their right visual field.

\section{Digit span}

Thirteen patients could not be tested on more than four occasions, usually due to aphasia; the remaining 49 patients were within the normal range (that is within the 95 th percentile) ${ }^{9}$ Recovery in these 49 patients was judged by taking the average of each patient's first two results from the average of their last two results. Two patients showed an increase of three or more in the number of digits recalled, and eight more showed an increase of two points. The original test scores of these 10 patients, four of whom had mild aphasia, are plotted in fig 3 .

\section{Word recall}

Eighteen patients could not be tested successfully on more than four occasions, leaving 44 patients whose recovery could be assessed. Taking the difference between the average of each patient's first two and last two scores as a measure of recovery, nine patients could recall $2 \cdot 0-4 \cdot 5$ more words by 3 months than on their first assessment. Two of these patients had shown recovery in digit span, and two were judged clinically by a speech therapist to have aphasia. Their original scores are plotted in fig 4 .

\section{Discussion}

This study of the recovery of cognitive functions after stroke is unusual in two ways. Firstly, it has included a wide spectrum of patients selected only by virtue of admission to hospital and survival for 3 months. Secondly, it involved repeated assessments over the first 3 months after the stroke, concentrating on this vital early period. This study has also provided some data from normal elderly people for a commonly used test of visual neglect. The results have demonstrated recovery from visual neglect in nearly one quarter of all patients over the first 3 months. There was probable improvement in attention and verbal recall in one fifth, although interpretation is complicated by the presence of coincident mild aphasia in some patients.

The population studied should be representative of most patients who are admitted to hospital, and survive 3 months. The patients had a wide range of deficits, and were not selected on the grounds of age, severity or any other criteria. Indeed this explains the high numbers who could not be assessed and probably underlies the variability seen in other patients attempting the cancellation task.

The investigation of recovery of cognitive functions after stroke is made difficult by the absence of suitable measures, standardised and validated in an elderly population, yet also short and sufficiently simple to beo used frequently over a few months. The tests used were chosen because they had been used in other stud- $\cap$ ies, and they were relatively short and simple. The digit span is well tested for reliability and normal performance in an elderly population. ${ }^{9}$ Visual cancellation tasks are commonly used. Published data from elderly people concerning their performance, and standardised scoring methods are not available. The scoring method used here warrants a brief discussion. Errors and omissions on the task are usually analysed in clinical practice, but this can pose problems. Poor concentration could lead to many omissions which might be scattered randomly. Clearly the total number of errors would not reflect neglect alone.

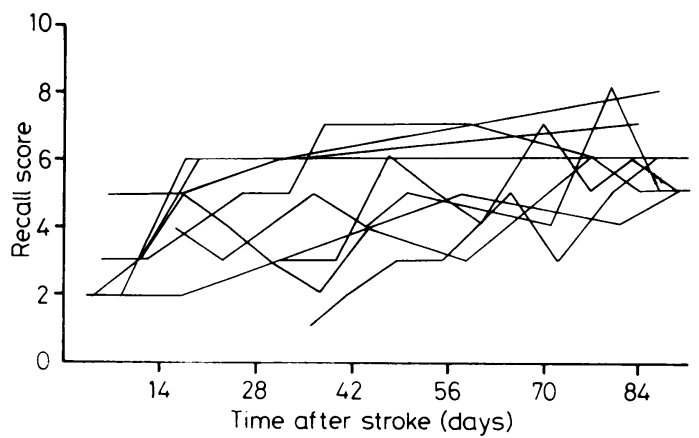

Fig 4 Recovery of verbal recall. 9 patients; number of words (from list of 10) correctly recalled. 
Therefore, a measure of the relative performance on right and left is needed, and we opted for the relative proportion of correct cancellations.

One particular problem in measuring recovery arises because the investigator rarely knows the patient's pre-stroke ability. Simply classifying people as normal or abnormal may hide considerable loss in individual patients. One possible solution is to use a patient's results from long after his stroke as the nearest estimate of their "normal" ability. This solution assumes that the stroke has not improved the patient's ability. One then assumes that any improvement seen after stroke implies that there was some loss at the time of stroke and that the patient is recovering towards his pre-stroke performance. Further, it assumes that the patient is not simply learning the test. As we did not use alternative forms of our tests, it is clearly possible that patients were just learning the test material but our reliability studies suggest that any learning was probably small. In our study the tests were separated by 2 week intervals and the patients were not told the correct answers.

The results of this study suggest that at least one quarter of patients who survived 3 months had some visual neglect initially. This affected either visual field and there was recovery to normal function within 70 days in these patients. We did not know how many people were left with visual inattention at 3 months as the assessments in a substantial number of patients were unreliable or impossible. Attention span was within the 95th percentile ("normal") in all testable patients. However, some improvement was shown by 10 patients, suggesting that they had suffered some loss relative to their pre-stroke ability. Verbal memory similarly seemed to improve in nine patients. The improvement in digit span and word recall may have been secondary to recovery from aphasia in some patients.

It is difficult to evaluate these results as there are no directly comparable studies. A recent study, based on Albert's test, ${ }^{10}$ found neglect in $49 \%$ of patients with non-dominant hemisphere lesions and $25 \%$ of patients with dominant hemisphere lesions. Interestingly, neglect was definitely absent in nearly equal proportions, the difference being that more patients with dominant hemisphere lesions were unassessable. Observations on patients from a community register of stroke found that $11 \%$ of all patients had some visual neglect, with neglect being more frequent $(14 \%)$ in patients with right sided symptoms; recovery was observed between 6 and 12 months. The recovery seen in neglect is similar to that found in a study restricted to patients with right hemisphere stroke. ${ }^{3}$ It has also been found, in a population based study on verbal and visual memory after stroke, that disturbance of memory was frequent, and that it recovered between 3 and 6 months in some patients. ${ }^{11}$

Two points should be made. First, the pattern of change seen in the present study is similar to that seen in other spheres such as arm function or dressing ability, with a rapid early phase of recovery. ${ }^{4}$ Second, there was considerable variation between patients.

Further understanding of the frequency of these losses soon after stroke and their recovery over the first few months will depend upon the development and use of more suitable tests. In particular the presence of aphasia complicates the administration and interpretation of most tests including cancellation tasks, even Albert's test which simply uses lines. ${ }^{10}$ Meanwhile we conclude that visual neglect is not uncommon after stroke; that many patients recover from visual neglect within 10 weeks; and that attention span and verbal memory is probably reduced in some patients immediately after stroke.

We thank all the physicians who allowed us to study their patients; Drs Gordon, Heller and Parker for their help; Dr A Sunderland, Mr C Skilbeck and Mrs J Legh-Smith for their advice; Mr Peter Cox for his artwork; the Bristol and Avon Stroke Foundation for financial support; and all the patients and normal controls for their cooperation.

\section{References}

1 Lendrem, W, Lincoln NB. Spontaneous recovery of language in patients with aphasia between 4 and 34 weeks after stroke. J Neurol Neurosurg Psychiatry 1985; 48:743-8.

2 Skilbeck CE, Wade DT, Langton Hewer R, Wood VA. Recovery after stroke. J Neurol Neurosurg Psychiatry 1983;46:5-8.

3 Meerwaldt JD. Spatial disorientation in right hemisphere infarction: a study of the speed of recovery. $J$ Neurol Neurosurg Psychiatry 1983;46:426-9.

4 Wade DT, Wood VA, Langton Hewer R. Recovery after stroke-the first three months. J Neurol Neurosurg Psychiatry 1985;48:7-13.

5 Aho K, Harmsen P, Hatano S, Marquardsen J, Smirnov VE, Strasser T. Cerebrovascular disease in the community: results of a WHO collaborative study. Bull WHO 1980;58:113-30.

6 Bhavnani G, Cockburn J, Whiting S, Lincoln N. The reliability of the RPA and implications for some commonly used assessments of perception. Br J Occup Ther 1983;46:17-19.

7 Diller L, Weinberg J. Hemi-inattention in rehabilitation: the evolution of a rational remediation program. In: Weinstein EA, Friedland RP (eds). Advances in Neurology. New York, Raven Press. 1977;18:63-82.

8 Lezak MD. Neuropsychological Assessment. New York, Oxford University Press. 1976.

9 Wechsler D. A standardised memory scale for clinical use. J Psychol 1945;19:87-95.

10 Fullerton KJ, McSherry D, Stout RW. Albert's test: a neglected test of perceptual neglect. Lancet 1986; i: $430-2$.

11 Wade DT, Parker V, Langton Hewer R. Memory disturbance after stroke: frequency and associated losses. Int Rehabil Med 1986;8:60-64. 